\title{
118 ANOS DE DADOS MENSAIS DO ÍNDICE PADRONIZADO DE PRECIPITAÇÃO: SÉRIE METEOROLÓGICA DE CAMPINAS, ESTADO DE SÃO PAULO
}

\author{
GABRIEL CONSTANTINO BLAIN ${ }^{1}$, MARY TOSHIE KAYANO ${ }^{2}$ \\ ${ }^{1}$ Instituto Agronômico de Campinas (IAC), Campinas, SP, Brasil \\ ${ }^{2}$ Instituto Nacional de Pesquisas Espaciais (INPE) \\ gabriel@iac.sp.gov.br, mary.kayano@cptec.inpe.br
}

Recebido Outubro 2009 - Aceito Junho 2010

\begin{abstract}
RESUMO
O Índice Padronizado de Precipitação (SPI) é utilizado em programas estaduais e nacionais no monitoramento das condições de seca de diversas regiões brasileiras. Com base na hipótese de que a análise de longas séries temporais do SPI pode auxiliar a adoção de políticas de mitigação e combate a essa anomalia climática, o objetivo desse trabalho foi analisar a variabilidade do SPI mensal, na localidade de Campinas-SP, entre os anos de 1890 a 2007. Por meio de análises espectrais e testes não paramétricos verificou-se uma variabilidade na escala de três a quatro anos. Contudo, não foi possível observar marcante influência do fenômeno El Niño/Oscilação Sul nas condições mensais de variabilidade climática na localidade de Campinas. Com respeito à tendência de longo prazo, enquanto uma tendência de intensificação nas condições de déficit de precipitação pluvial foi detectada em agosto, nos demais meses não houve alterações significativas. Sob o ponto de vista acadêmico o tratamento probabilístico e padronizado dos déficits/excesso de precipitação pluvial empregado no cálculo do SPI, o torna um interessante índice alternativo na investigação de forçantes climáticas condicionantes/moduladoras do clima de determinada região.
\end{abstract}

Palavras Chave: variabilidade temporal, SPI, seca

\begin{abstract}
YEARS OF MONTHLY STANDARDIZED PRECIPITATION INDEX DATA: METEOROLOGICAL SERIES OF CAMPINAS, STATE OF SÃO PAULO

The Standard Precipitation Index (SPI) is used in state and national monitoring programs of the drought conditions in several Brazilian regions. Based on the hypothesis that the analysis of long term SPI time series might help on the adoption of policies of mitigation and facing climate anomalies, this work aims to analyze the variability of monthly SPI, in Campinas (SP) during the years from 1890 to 2007. From spectral analyses and non-parametric tests, a variability of three to four years scale was noted for this index. However, a remarkable influence of the El Niño/Southern Oscillation on the variability of monthly climate conditions in Campinas was not seen. Concerning the long term trends, while a strengthening trend of rainfall deficit was noted during August, no significant alterations was noted for the other months. Under the academic point of view, the probabilistic and standard treatment of deficit/excess of rainfall used in the SPI calculation makes it an interesting alternative index in investigating the conditioning/modulating climatic forcing of the climate of a given region.
\end{abstract}

Keywords: temporal variability, SPI, drought 


\section{INTRODUÇÃO}

Dentre as adversidades meteorológicas que mais afetam o ser humano, a seca ocupa posição de destaque. As dificuldades associadas à mitigação e combate dos efeitos dessa anomalia climática têm início desde o estabelecimento de uma definição clara do termo seca, até o conhecimento da vulnerabilidade ${ }^{1}$ que cada atividade, região ou sociedade apresenta em relação a essa condição climática adversa. Nesse aspecto, Wilhite et al. (1987) afirmam que o estudo da seca não deve ser separado do contexto social. Contudo, para esses autores, todos os casos desse fenômeno são originados por um déficit de precipitação pluvial, que resulta em baixa disponibilidade hídrica para a atividade que a requer.

Sob as diversas abordagens que as avaliações dessa adversidade podem ser conduzidas, entre elas a agrícola, a hidrológica ou até mesmo a sócio-econômica, o enfoque meteorológico, ou simplesmente a seca meteorológica, é relacionada, por autores como Wilhite (2000), a um déficit de precipitação. Em outras palavras, esse último tipo de seca ocorre quando os totais de precipitação pluvial observados em determinada região e em um período específico, encontram-se consideravelmente abaixo do que seria climatologicamente esperado.

O Índice Padronizado de Precipitação(SPI), desenvolvido por McKee et al. (1993), vem sendo utilizado em programas estaduais e nacionais no monitoramento das condições de seca de diversas regiões brasileiras; entre esses, os portais INFOSECA ${ }^{2}$, da Secretaria de Agricultura e Abastecimento do Estado de São Paulo, e o Instituto Nacional de Meteorologia ${ }^{3}$, do Ministério da Agricultura, Pecuária e Abastecimento, utilizam esse índice na quantificação padronizada dos déficits de precipitação pluvial (PRE), evidenciando regiões submetidas a condições severas dessa adversidade climática.

$\mathrm{O}$ algoritmo de cálculo utilizado na estimação desse índice de seca, bem como diversas considerações a respeito de possíveis usos/aplicações desse índice de seca podem ser encontradas em McKee et al. (1993 e 1995), Guttman (1998 e 1999), Hayes et al. (1999), Heim Junior (2002), entre outros. Tadesse et al. (2005), utilizando o SPI na caracterização dos episódios de seca ocorridos na região de Nebraska, EUA, entre 1950 e 1999, indicam que a maioria dos casos dessa adversidade meteorológica foi precedida por valores negativos do Índice de Oscilação Sul (IOS). Para Zhai et al. (2010) o SPI pode ser usado para descrever tendências climáticas nas condições de

1 Grau que um sistema é susceptível, ou incapaz de enfrentar efeitos adversos. É uma função entre a natureza, a magnitude e o percentual de uma variação que o sistema é exposto, sua sensitividade, sua capacidade adaptativa. Depende, ainda, de uma relação complexa de situações econômicas, sociais, culturais e políticas, que são configuradas por eventos extremos já enfrentados por determinadas sociedades (IPCC, 2001)

$2 \quad$ www.infoseca.sp.gov.br

3 http://www.inmet.gov.br seca/excesso de umidade na China ou nas demais regiões do globo terrestre.

No território brasileiro, Li et al. (2008) indicam tendência de queda nos valores do SPI, relativos ao sul da região Amazônica, entre os anos de 1970 e 1999. Para o estado de São Paulo, apesar dos trabalhos de Sansigolo (2004), que estabelece uma análise comparativa entre o desempenho do índice de seca de Palmer (Palmer, 1965) e do SPI na localidade de Piracicaba (1917 a 2001) e o de Blain (2005), que avalia a aplicação do SPI nas condições climáticas do estado, relacionando-o a parâmetros do balanço hídrico climatológico de Thorntwaite e Mather (1955); a investigação de tendências climáticas bem como de possíveis relações entre a variabilidade temporal desse índice e fenômenos de grande escala, tais como o El Niño/Oscilação Sul (ENOS), parecem ser ainda pouco exploradas.

Dessa forma, o objetivo desse trabalho é analisar a variabilidade da série temporal do SPI mensal, na localidade de Campinas-SP, entre os anos de 1890 a 2007. Tendências climáticas, localização temporal de possíveis picos de variância e relação com o fenômeno El Niño/Oscilação Sul foram investigadas. Espera-se que este trabalho agregue importantes informações à literatura científica relativa à análise, mitigação e monitoramento do fenômeno seca.

\section{MATERIAL E MÉTODO}

Foram utilizados dados de precipitação pluvial mensal

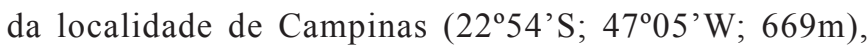
pertencente ao Instituto Agronômico (IAC/APTA/SAA) entre os anos de 1890 a 2007. Essa série meteorológica tem sido utilizada em diversos trabalhos, tais como Blain (2005), Blain et al. (2007), Kayano e Blain (2007), Blain et al (2009) e Blain (2009) que comprovam, utilizando análises paramétricas, não paramétricas e espectrais, a consistência da mesma. De acordo com a classificação de Köeppen, o clima desse município é Cwa (temperatura média do mês mais quente maior ou igual à $22^{\circ} \mathrm{C}$, do mês mais frio menor que $18^{\circ} \mathrm{C}$ e precipitação média do mês mais seco menor que $30 \mathrm{~mm}$ ). Na área atuam tanto sistemas tropicais, dos quais o tropical atlântico é o mais importante em termos de incidência temporal, como polares, com destaque para o polar atlântico, que de acordo com Monteiro (1973) comanda o ritmo climático regional (Nunes, 1997).

$\mathrm{Na}$ escala mensal, o cálculo do SPI inicia-se com a determinação da probabilidade acumulada de ocorrência $\mathrm{H}\left(\mathrm{X}_{\mathrm{t}, \mathrm{i}}\right)$, para $1 \leq \mathrm{t} \leq 12$ e i $\in \mathrm{Z}$, associada aos dados de precipitação pluvial $\left(\mathrm{X}_{\mathrm{t}, \mathrm{i}}\right)$. Essa probabilidade é, no estado de São Paulo, usualmente estimada com base na distribuição gama $\mathrm{G}\left(\mathrm{X}_{\mathrm{t}, \mathrm{i}}\right)$ com dois parâmetros, conforme exemplificado em Thom (1966), Blain et al. (2007) e Blain et al. (2009). A expressão matemática dessa distribuição paramétrica pode ser encontrada em diversos 
trabalhos, entre eles Wilks (2006). Os parâmetros de forma $(\alpha)$ e de escala ( $\beta$ ) são freqüentemente estimados com base no método da máxima verossimilhança. É importante ressaltar que a distribuição gama é indefinida para $\mathrm{x}=0$. Dessa forma, $\mathrm{H}\left(\mathrm{X}_{\mathrm{t}, \mathrm{i}}\right)$ é descrita por:

$$
H\left(X_{t}\right)=q+(1-q) G(x) \text { para } q=(m / N)
$$

Em que $\mathrm{m}$ e $\mathrm{N}$ são, respectivamente, o número de observações $X_{t}=0$ e o número de dados de cada série mensal. $\mathrm{H}\left(\mathrm{X}_{\mathrm{t}}\right)$ é então transformada em uma variável normal, valor final do SPI, utilizando-se as equações desenvolvidas por Abramowitz e Stegun, (1965).

$$
\begin{aligned}
& S P I=-\left(t-\frac{o+c_{1} t+c_{2} t^{2}}{1+d_{1} t+d_{2} t^{2}+d_{3} t^{3}}\right) \text { para } 0<\mathrm{H}(\mathrm{x}) \leq 0,5 \\
& S P I=+\left(t-\frac{o+c_{1} t+c_{2} t^{2}}{1+d_{1} t+d_{2} t^{2}+d_{3} t^{3}}\right) \text { para } 0,5>\mathrm{H}(\mathrm{x})<1
\end{aligned}
$$

com

$$
\begin{aligned}
& t=\sqrt{\left(\ln \left(\frac{1}{\left(H(x)^{2}\right.}\right)\right.} \quad \text { para } 0<\mathrm{H}(\mathrm{x}) \leq 0,5 \\
& t=\sqrt{\left(\ln \left(\frac{1}{\left(1-H(x)^{2}\right.}\right)\right.} \quad \text { para } 0,5>\mathrm{H}(\mathrm{x})<1
\end{aligned}
$$

$c_{o}=2,515517 ; c_{1}=0,802853 ; c_{2}=0,010328 ; d_{1}=1,432788 ; d_{2}=0,189269 ; d_{3}=0,001308$

Segundo Hayes et al. (1999) por se ajustarem à distribuição normal, pode-se esperar em uma longa série histórica, que $68 \%$ dos valores do SPI estejam entre 1 e -1 , $95 \%$ dos valores estejam entre 2 e -2 e $99 \%$ dos valores estejam entre 3 e -3. Uma interpretação relacionada é que magnitudes do SPI menores que -1 ocorram dezesseis vezes em cem anos, magnitudes do SPI menores que -2 ocorram de duas a três vezes em cem anos e valores menores que menos três ocorram uma vez a cada duzentos anos. Como conseqüência direta dessa última característica, o SPI não apresenta a habilidade de detectar regiões mais sujeitas à seca (Hayes et al., 1999). O evento seca começa quando o SPI torna-se negativo e atinge o valor de -1 e termina quando este volta a apresentar valores positivos. $\mathrm{Na}$ Tabela 1 são ilustradas as categorias de seca, de acordo com o SPI, descritas por McKee et al. (1993).

A função autocorrelação (acf), conforme descrito em Wilks (2006), foi aplicada a fim de verificar o grau de persistência temporal da série do SPI mensal. Tendo em vista que a principal característica da PRE mensal no estado de São Paulo é sua elevada variabilidade temporal (Blain, 2009), é esperado que os coeficientes da acf permaneçam dentro do limite do ruído branco, indicando que a probabilidade de ocorrência de um dado SPI, estimado no i-ésimo mês, seja independente dos valores de $\mathrm{SPI}_{\mathrm{i}-\mathrm{j}}$, para j $\varepsilon$ Z. Para toda a série estimou-se a acf até o lag 36 , que neste caso corresponde à defasagem de 36 meses. Para a aplicação da acf em cada série mensal os coeficientes de autocorrelação foram estimados até o lag 6 (defasagem de 6 anos). O número de lags foi arbitrariamente escolhido, respeitando-se, entretanto, o limite j/4 para sua estimação, em que j é o número total de dados analisados.

A análise de ondaleta foi empregada a fim de determinar a existência de possíveis picos de variância presentes na série do SPI, bem como a localização temporal dos mesmos. Esse mesmo método foi aplicado nos resíduos mensais de PRE. Essa última é estimada por meio da subtração entre Xt e sua respectiva mediana mensal.

Para Guedes et al. (2005), a transformada de Fourier (TF) é comumente utilizada no estudo das periodicidades de séries geofísicas. $\mathrm{Na} \mathrm{TF}$, a série é considerada estacionária e as periodicidades presentes em toda a série são obtidas. Entretanto, com o uso da transformada de ondaleta (TO), a decomposição é realizada não somente na freqüência, como na TF, mas também no tempo, ou seja, a TO produz um espectro de potência decomposto em tempo e escala (freqüência) denominado de potência local da ondaleta (WPS; local wavelet power spectrum). Com isso, a TO permite tanto caracterizar a intermitência dos sinais geofísicos, quanto indicar possíveis

Tabela 1 - Valores do Índice Padronizado de Precipitação (SPI) e Categorias de seca ou de umidade.

\begin{tabular}{|c|c|}
\hline SPI & Categoria \\
\hline$\geq 2, \mathrm{OO}$ & Extremamente Úmido \\
\hline 1,50 a 1,99 & Severamente Úmido \\
\hline 1,00 a 1,49 & Moderadamente Úmido \\
\hline 0,10 a 0,99 & Umidade Incipiente \\
\hline 0,00 a $-0,99$ & Seca Incipiente \\
\hline$-1,00$ a $-1,49$ & Moderadamente Seco \\
\hline$-1,50$ a $-1,99$ & Severamente Seco \\
\hline$\leq-2,00$ & Extremamente Seco \\
\hline
\end{tabular}


interações entre os picos de variância em diversas escalas temporais (Guedes et al., 2005). A média temporal do espectro de potência em tempo-escala é denominada de espectro de potência global (GWP). Nakken (1998) afirma que análise de ondaleta é um método importante para investigar características não-estacionárias em séries de precipitação e de escorrimento superficial, revelando mudanças abruptas, pontos de quebra e descontinuidades nos sinais desses elementos que a TF não é capaz de revelar. Segundo Morettin (1999), na análise de Fourier, toda função periódica, de período $2 \pi, \mathrm{L}^{2}(0,2 \pi)$, é gerada por superposição de exponenciais complexas $\mathrm{Wn}(\mathrm{x})=\mathrm{e}^{\mathrm{inx}}$, para $\mathrm{n} \in \mathrm{Z}$, obtidas por dilatação da função $\mathrm{w}(\mathrm{x})$. Na análise de ondaletas, essa idéia é estendida à $\mathrm{L}^{2}(\mathrm{R})$, gerando o novo espaço com base em uma função padrão, a ondaleta Mãe $(\psi)$.

De acordo com Nakken (1998), a ondaleta mãe utilizada deve ter um padrão semelhante ao sinal em estudo. Sob o mesmo aspecto, Torrence e Compo (1998) afirmam que tipos de ondaletas mães como a de Morlet, são indicadas para o estudo de séries temporais com características oscilatórias sendo, com isso, apropriadas para analisar a amplitude e a fase do sinal sob avaliação. Dessa forma, a função ondaleta mãe adotada foi a de Morlet (Equação 4). Autores como Torrence e Compo (1998), Torrence e Webster (1999), Nakken (1998), Reboita (2004), Guedes et al. (2005), Kayano e Blain (2007) e Blain (2009) utilizam essa ondaleta mãe na análise de séries temporais de PRE.

$$
\psi(\eta)=\pi^{-1 / 4} e^{i 6 \eta} e^{-\frac{1}{2} \eta^{2}}
$$

Em que: $\eta$ refere-se ao período ou escala temporal de análise (adimensional)

Autores como Torrence e Compo (1998) e Guedes et al. (2005) descrevem que a ondaleta de Morlet pode ser entendida como uma função periódica cuja amplitude é modulada por uma gaussiana. A transformada contínua da ondaleta (CWT) é descrita, para uma série $\mathrm{x}_{\mathrm{n}}$, com $\mathrm{n}=1$ :j e dados constantemente espaçados por $\delta t$, pela expressão 5 .

$$
\left.W_{n}^{X}(s)=\sqrt{\frac{\delta t}{s}} \sum_{n^{\prime}=1}^{j} x_{n^{\prime}} \psi_{o}\left[n^{\prime}-n\right) \frac{\delta t}{s}\right]
$$

Em que $\mathrm{W}$ é o resultado da transformação contínua de ondaleta do sinal $\mathrm{x}(\mathrm{t})$.

Em Reboita (2004) a descrição completa desse método e um excelente exemplo hipotético são ilustrados. Considerando duas séries temporais: $\mathrm{s}(\mathrm{t})=\operatorname{sen}(10 \mathrm{t})+\operatorname{sen}(20 \mathrm{t})$ e $\mathrm{g}(\mathrm{t})=\operatorname{sen}(10 \mathrm{t})$ seguido por sen(20t), Reboita (2004) demonstra a habilidade da análise de ondaletas em detectar alterações, no espaço tempo-freqüência, nos picos de potência de uma série temporal. No presente estudo, a análise de ondaletas foi aplicada utilizando o algoritmo elaborado por Torrence e Compo (1998) na linguagem utilizada pelo software Matlab e disponível em http://paos.colorado.edu/research/wavelets.
A determinação do nível de significância dos picos espectrais, tanto na análise de Fourier quanto na de ondaleta, exige a escolha de um espectro de fundo apropriado. Nessa decisão, assume-se que diferentes realizações de um processo geofísico distribuem-se aleatoriamente ao redor desse valor espectral esperado (Torrence e Compo 1998). Para diversos fenômenos físicos, esse espectro de fundo pode ser baseado em processos de ruído branco ou vermelho. De acordo com Torrence e Compo (1998), Torrence e Webster (1999) e Grinsted et al. (2004), entre outros, a Ho pode então ser definida por meio do seguinte algoritmo em que a série temporal apresenta um espectro médio dado pela Equação 6.

$$
\begin{aligned}
P k & =\frac{1-\alpha}{1+\alpha^{2}-2 \alpha \cos (2 \pi k / j)} \\
\operatorname{para} \mathrm{K} & =0 \ldots \mathrm{j} / 2 \mathrm{e} \\
\alpha & =\frac{r_{1}+\sqrt{r_{2}}}{2}
\end{aligned}
$$

Em que $r_{1}$ e $r_{2}$ são os coeficientes da acf para as defasagens (lags) 1 e 2 da série com j dados. Na Equação 6 é possível verificar que se a série sob análise for oriunda de um processo de ruído branco, Pk será constante para todo $\mathrm{k}$. A distribuição de nulidade para a WPS, associada à Ho, pode então ser descrita pela expressão 7.

$$
\left|\frac{W_{n}(S)}{\sigma^{2}}\right|^{2}=\frac{1}{2} P_{k} \chi_{2}^{2}
$$

Em que: $\sigma^{2}$ é a variância da série temporal; $\chi_{2}^{2}$ representa a distribuição qui-quadrado com dois graus de liberdade.

Torrence e Compo (1998), Torrence e Webster (1999) e Grinsted et al. (2004), entre outros, utilizam o nível de significância de $5 \%$, ou percentil 0,95 da $\chi_{2}^{2}$,para a condução do teste de hipótese.

Autores como Torrence e Webster (1999) e Grinsted et al. (2004) indicam que a coerência das CWT de duas séries temporais X e Y pode ser descrita por:

$$
R_{n}^{2}(s)=\frac{\mid S\left(\left.s^{-1} W_{n}^{X}(s)\right|^{2}\right.}{S\left(s^{-1}\left|W_{n}^{X}(s)\right|^{2}\right) S\left(s^{-1}\left|W_{n}^{y}(s)\right|^{2}\right)}
$$

Em que S é um operador de suavização.

A Equação 7 pertence à forma de investigação estatística denominada de análise cruzada de ondaleta. De acordo com Grinsted et al. (2004), é interessante interpretar a Equação 8 como sendo o coeficiente de correlação de Pearson, localizado no espaço tempo-freqüência. A descrição completa desse método é dada em trabalhos como os de Grinsted et al. (2004). A análise 
cruzada de ondaleta foi aplicada considerando-se as séries do SPI e do Índice de Oscilação Sul.

Segundo Reboita (2004), a Oscilação Sul foi pioneiramente observada por Sir Gilbert Walker em 1924 ao notar a alternância das condições da pressão atmosféricas ao nível médio do mar (PNM) entre as localidades de Darwin, Australia $\left(12^{\circ} \mathrm{S}\right.$ e $\left.130^{\circ} \mathrm{E}\right)$ e do Tahiti $\left(17^{\circ} \mathrm{S}\right.$ e $\left.150^{\circ} \mathrm{W}\right)$. O IOS é uma medida da diferença de PNM entre essas duas regiões. Valores negativos do IOS caracterizam eventos de El Niño, ao passo que valores positivos caracterizam episódios de La Niña. O termo El Niño refere-se ao aumento da temperatura da superfície do mar (TSM) no Pacífico tropical, ao passo que o termo La Niña refere-se ao resfriamento da TSM. Diversos trabalhos tais como, Ropelewsky e Halpert (1987 e 1989), Mello et al. (1999a e 1999b), Cunha et al. (1999 e 2001) e Minuzzi et al. (2007), procuraram relacionar o fenômeno ENOS à variabilidade temporal de elementos meteorológicos, utilizando o IOS como indicador da magnitude desse fenômeno. Os dados do IOS, disponíveis em http://www.bom.gov.au/climate/current/ soihtm1.shtml, foram, na escala mensal, calculado por:

$$
\text { IOS }=10 *[\text { Pdiff }- \text { Pdiffmed }] / \text { DP(Pdiff) }
$$

Em que:

Pdiff: Diferença entre a Pressão atmosférica ao nível do mar, observada nas localidades de Darwin e Tahiti;

Pdiffmed: Média aritmética mensal (1933 a 1992) dos dados de Pdiff;

DP(Pdiff): Desvio Padrão mensal (1933 a 1992) dos dados de Pdiff;

O teste não paramétrico denominado de Mann-Kendall Sazonal (MK) foi empregado nas séries mensais do SPI. Inicialmente proposto por Mann (1945) e Kendall e Stuart (1967) esse teste pode ser definido, para uma série $\mathrm{Z}=\{\mathrm{Z} 1, \mathrm{Z2}, \ldots \mathrm{Zi}, \mathrm{Zj}, \ldots . . \mathrm{Zn})$ por:

$$
\mathrm{T}=\sum_{\mathrm{j}<\mathrm{i}} \operatorname{sinal}\left(\mathrm{Z}_{\mathrm{i}}-\mathrm{Z}_{\mathrm{j}}\right)
$$

em que:

$$
\operatorname{sinal}\left(Z_{\mathrm{i}}-Z_{\mathrm{j}}\right)=\left\{\begin{array}{l}
1 \text { para } Z_{\mathrm{i}}-Z_{\mathrm{j}}>0 \\
0 ; \text { para } \mathrm{Z}_{\mathrm{i}}-\mathrm{Z}_{\mathrm{j}}=0 \\
-1 ; \text { para } \mathrm{Z}_{\mathrm{i}}-\mathrm{Z}_{\mathrm{j}}<0
\end{array}\right\}
$$

Considerando a independência temporal (ruído branco) entre os n valores da série; quando não há presença de tendências $\mathrm{T}$ é normalmente distribuído com média $\mathrm{E}(\mathrm{T})=0$ e variância $\operatorname{Var}(\mathrm{T})$. O valor final da estatística MK pode ser estimado por:

$$
\mathrm{MK}=\left\{\begin{array}{l}
\frac{\mathrm{T}-1}{\sqrt{\operatorname{Var}(\mathrm{T})}} ; \text { para } \mathrm{T}>0 \\
0 ; \text { para } \mathrm{T}=0 \\
\frac{\mathrm{T}+1}{\sqrt{\operatorname{Var}(\mathrm{T})}} ; \text { para } \mathrm{T}<0
\end{array}\right\}
$$

$$
\operatorname{Var}(T)=\frac{n(n-1)(2 n+5)}{18}
$$

Adotando-se como Ho a inexistência de tendências temporais; essa hipótese não é rejeitada se, em um teste bilateral, o valor absoluto de MK for menor que um determinado valor da tabela da distribuição normal reduzida. Autores como Marengo et al. (2007) utilizam o nível de significância $\alpha=0,05$. Adotando-se esse mesmo nível, Ho não será rejeitada sempre $-1,96<\mathrm{MK}<1,96$. Para o nível $\alpha=0,10$ Ho não será rejeitada sempre $-1,67<\mathrm{MK}<1,67$. Tendências de elevação ou queda resultam em valores positivos ou negativos de MK. Esse método foi aplicado separadamente em cada uma das 12 séries mensais, em que o intervalo entre dois valores adjacentes é de 1 ano, e em toda a série, em que o intervalo entre dois dados é de 1 mês.

\section{RESULTADOS E DISCUSSÃO}

\subsection{Considerações iniciais}

Considerando o algorítmo de estimação do SPI, verifica-se que quando $X_{t, i}=0$, o valor final do índice de seca é dado ou limitado pela probabilidade empírica q (Equação 1). Com isso, nos meses em que o valor zero de precipitação pluvial apresenta uma probabilidade de ocorrência relativamente elevada (por exemplo, $\mathrm{q}=\mathrm{H}(\mathrm{x})=0,20$; Equação 1), o menor valor possível do SPI (associado à $\mathrm{PRE}=0$ ) também será relativamente elevado $(\mathrm{H}(\mathrm{x})=0,2$ resulta em $\mathrm{SPI}=-0,80$; equações 2 e 3 ). Essa característica intrínseca do modelo SPI vai ao encontro da definição de seca meteorológica, dada por Wilhite (2000), como resultante de um déficit de precipitação pluvial. Em regiões e períodos em que o valor de precipitação $\left[X_{t, i}=0\right]$ possui freqüência de ocorrência relativamente elevada, esse mesmo valor não representa, sob o ponto de vista de um modelo probabilístico, um severo desvio na série temporal sob estudo.

Para uma dada série temporal, independentemente do mês sob estudo a aplicação das equações 2 e 3 garante que valores negativos do SPI representem dados de PRE com probabilidades acumulada de ocorrência inferiores a 0,5. Valores do SPI contidos no intervalo [-0,5 a 0,5$]$ indicam que o total de precipitação observado em um i-ésimo mês situa-se, aproximadamente, na parte central da distribuição, isto é, com probabilidades acumuladas entre 0,30 e 0,70. Essa relação é ilustrada na Figura 1.

Analisando a Figura 1, é possível verificar a característica, inerente ao SPI, no sentido de padronizar os excessos ou déficits de PRE, mesmo quando são comparadas curvas de probabilidade com forma bastante distintas. A Figura 1a é relativa à uma distribuição próxima à gaussiana, ao passo que a Figura 1e é associada à uma distribuição exponencial (parâmetro de forma da distribuição gama inferior à 1). Mesmo neste caso, um dado 
valor de SPI sempre representa, em termos de probabilidade de ocorrência, uma mesma condição climática. Comparando as Figuras 1a (1b) à 1d (1e) verifica-se também, que um dado valor de resíduo mensal de precipitação pluvial pode estar associado, sob o ponto de vista de probabilidades de ocorrências, à condições de déficit/excesso de PRE bastante distintas nessas duas curvas mensais de probabilidade. A capacidade de tornar comparável déficits/excessos de PRE oriundos de distribuições com formas e escalas consideravelmente distintas, corrobora Guttman (1999) que afirma que o SPI é um indicador de seca padronizado no tempo e no espaço. Nesse sentido, sob o aspecto teórico, o algoritmo de cálculo do SPI, descrito nas Equações 1 a 3 , e as interpretações relativas à Figura 1 podem ser extrapoladas para demais localidades em que os dados mensais de PRE provenham de uma população com distribuição gama.

\subsection{Análises estatísticas}

Na Figura 2 é ilustrada a acf da série do SPI, considerando todos os dados mensais temporalmente ordenados. $\mathrm{Na}$ aplicação da acf em séries meteorológicas mensais, compostas por dados Xt (pata $\mathrm{t}=1 \ldots 12$ ), é comum a obtenção do resíduo/ruído por meio da subtração das respectivas médias ou medianas mensais de cada valor de Xt (remoção da climatologia ou sazonalidade). Entretanto, conforme descrito, o SPI é ajustado à distribuição normal inversa padrão, ou seja, longas séries temporais desse índice de seca apresentarão médias próximas à 0 e variâncias próximas à unidade. Os 118 anos de dados mensais do SPI,
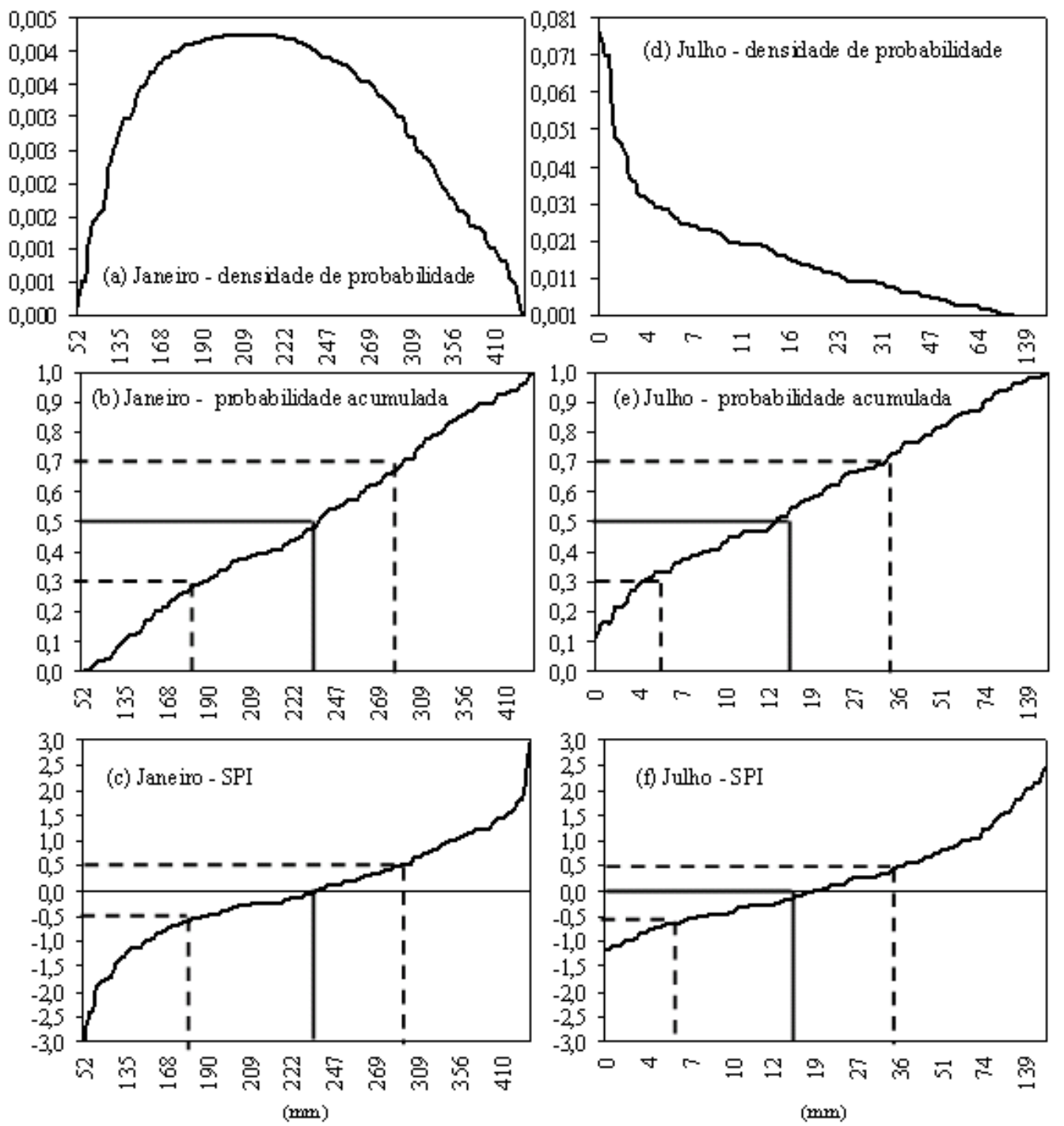

Figura 1 - a) Função densidade de probabilidade gama, probabilidades acumuladas e valores do SPI associados aos dados mensais de janeiro e Julho, na localidade de Campinas, SP (1890 a 2007). Os parâmetros de forma e escala são, respectivamente 6,1 e 40,9mm (janeiro) e 0,7 e $39,9 \mathrm{~mm}$ (Julho). O eixo das ordenadas é admensiomal 
utilizados no estudo tem média aritmética igual à 0,02 e desvio padrão de 0,98 . Com isso, este índice de seca pode também ser interpretado como o resíduo padronizado de totais de PRE observados em dada localidade.

$\mathrm{Na}$ Figura 2, verifica-se que a série do SPI mensal pode ser considerada, à 5\% de significância, oriunda de um processo de ruído branco. Essa característica pode ser vista, na localidade de Campinas, como uma indicação estatística da afirmação de Wilhite (2000) de que uma seca meteorológica pode ter seu início/fim de forma abrupta, uma vez que a probabilidade de ocorrência associada a um dado SPI mensal é independente dos valores desse índice observados em meses precedentes.

Nas Figuras 3 e 4 são ilustrados os espectros de potência da ondaleta das séries mensais de PRE (resíduo) e do SPI, respectivamente.

A Figura 3 é bastante semelhante à elaborada por Kayano e Blain (2007) para a série de residual de PRE de Campinas (1890 a 2006). Apesar de esses autores terem estimado o resíduo dos valores mensais de PRE subtraindo-se as respectivas médias aritméticas mensais (no presente estudo essa operação baseou-se nas medianas mensais), as conclusões de Kayano e Blain (2007) podem ser estendidas à Figura 3.

A potência global da ondaleta (GWP) da PRE exibe vários picos não significativos em torno de 2 a 8 anos e um mais acentuado com período próximo a quatro anos, resultante de potências (ou variâncias) significativas localizadas em alguns intervalos de tempo, como em torno de 1935, 1965 e 1980 (Figuras 3a e 3b). Na Figura 3c, que indica a média por escalas das potências ou variâncias (MEPO), verifica-se que, para a escala de 2-7 anos, alguns máximos valores de MEPO coincidem com anos de La Niña, como em 1908-10, 1933-34 e 1970-71. Porém, em outras La Niñas fortes (1955-56, 1973-74, $1975-76$ e 1988-89), a MEPO não mostra valores altos. Portanto, assim como indicado por Kayano e Blain (2007) e Blain et al. (2009) o sinal do ENOS na PRE da localidade de Campinas não é claro. Esse último resultado é também coerente com as observações de Rao e Hada (1994), para a região sudeste do Brasil, que observam que a influência do ENOS na variabilidade temporal da PRE não é conclusiva. Os máximos de MEPO nas escalas de 0,25-1 e de 2-7 anos em 1933-34 e 1970-71 são coincidentes. Essa característica pode indicar uma possível modulação de fenômenos de alta freqüência pelos de escala interanual (Kayano e Blain, 2007).

Diferentemente do observado para o resíduo da PRE mensal, a potência global da análise de ondaleta do SPI (Figura 4b), mostra um pico significativo de variância com período aproximado de 3 a 4 anos. Esse pico é fundamentalmente devido às potências localizadas nos anos de $1920 \mathrm{a} 1940 \mathrm{e} 1970 \mathrm{a} 1990$. Essa diferença pode ser vista como uma conseqüência direta das informações ilustradas na Figura 1, ou seja, um mesmo valor de SPI sempre representa a mesma condição de déficit/excesso de PRE, independentemente do mês de ocorrência. Contudo, não há garantias que essa última característica possa ser estendida a um mesmo valor residual de precipitação, tendo em vista a marcante alteração nas formas/escalas das curvas mensais de PRE (Blain, 2009) na localidade de Campinas. Com isso, torna-se possível inferir que o SPI constitui-se em uma interessante alternativa para a análise/avaliação da influência de fenômenos/fatores físicos, tais como o ENOS, na variabilidade espaço-temporal da precipitação pluvial de dada região, especialmente naquelas onde suas influências são mais marcadas como, por exemplo, nas regiões Sul e Nordeste do Brasil. Dessa forma, ainda que apenas sob o ponto de vista acadêmico/científico, torna-se relevante comparar a variabilidade temporal de índices relacionados ao ENOS, tais como o IOS à do SPI mensal. Na Figura 5, é ilustrada a coerência e diferença de fase do IOS e do SPI obtidas da análise cruzada de ondaleta (WTC).

Na Figura 5 é possível comprovar a dificuldade em estabelecer relação conclusiva entre a variabilidade temporal do ENOS e as condições mensais de precipitação na região de Campinas. Coerências significativas ao nível de $5 \%$ e superiores a 0,8 na escala do ENOS (2-8 anos) ocorrem em alguns curtos intervalos ao longo dos 117 anos analisados. Este é o caso, por exemplo, dos períodos em torno de 1905-1910, 1930, 1965 e de 1980-2000. Considerando estes períodos não há um padrão comum do ângulo de fase entre as duas séries.

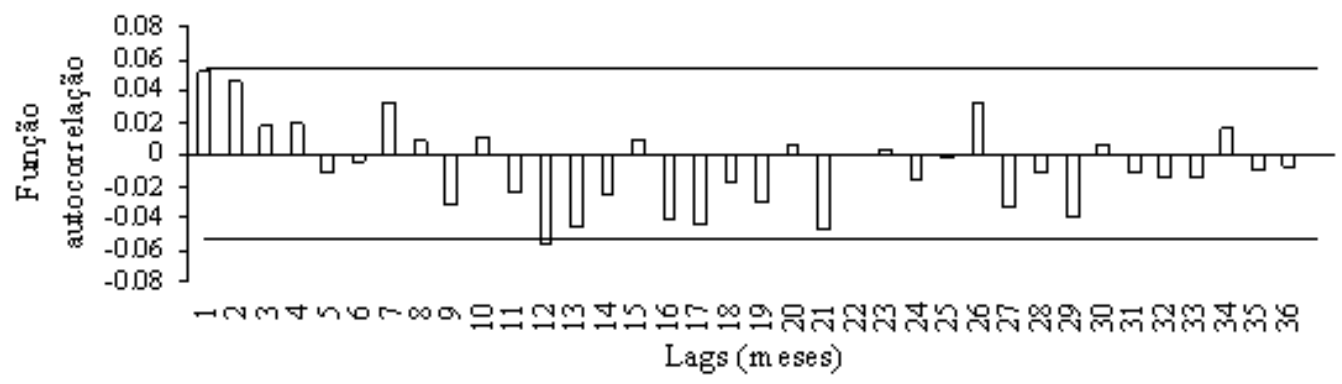

Figura 2 - Função autocorrelação aplicada à série mensal do Índice Padronizado de Precipitação (SPI) em Campinas, Estado de São Paulo (1890 a 2007). 
Para alguns períodos a diferença de fase é próxima de zero (1965) e para outros esta em torno e 180 graus. Nesse ponto, é interessante ressaltar que dois mecanismos ou processos físicos correlacionados, ao serem submetidos à análise como a descrita na Equação 7, tendem a apresentar ângulos de fase constantes em diversas escalas temporais (Grinsted et al., 2004). Essa indicação não pôde ser observada na Figura 5.

$\mathrm{Na}$ Tabela 2 é ilustrada a aplicação do MK nas séries do SPI de cada mês. Os coeficientes da acf relativas a cada série mensal também são ilustrados.
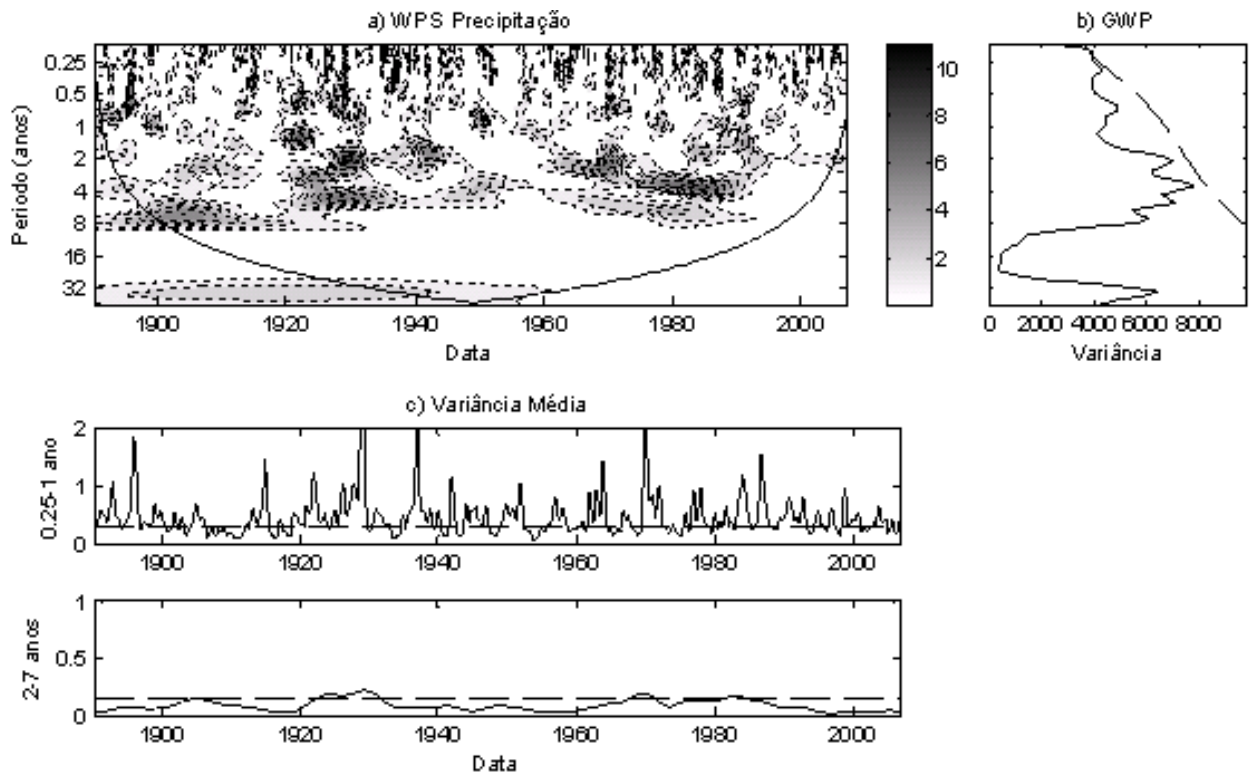

Figura 3 - a) Espectro de energia local da ondaleta para a precipitação pluvial residual mensal de Campinas-SP. Contornos tracejados correspondem a valores de variância normalizados variando de 1 a 10, conforme representado na barra horizontal. Contornos sombreados englobam áreas com variâncias significativas ao nível de $95 \%$ de confiança. A curva em forma de U representa o cone de influência, sob a qual o efeito de borda é importante; b) Espectro global de ondaleta; c) média por escalas das potências observadas.
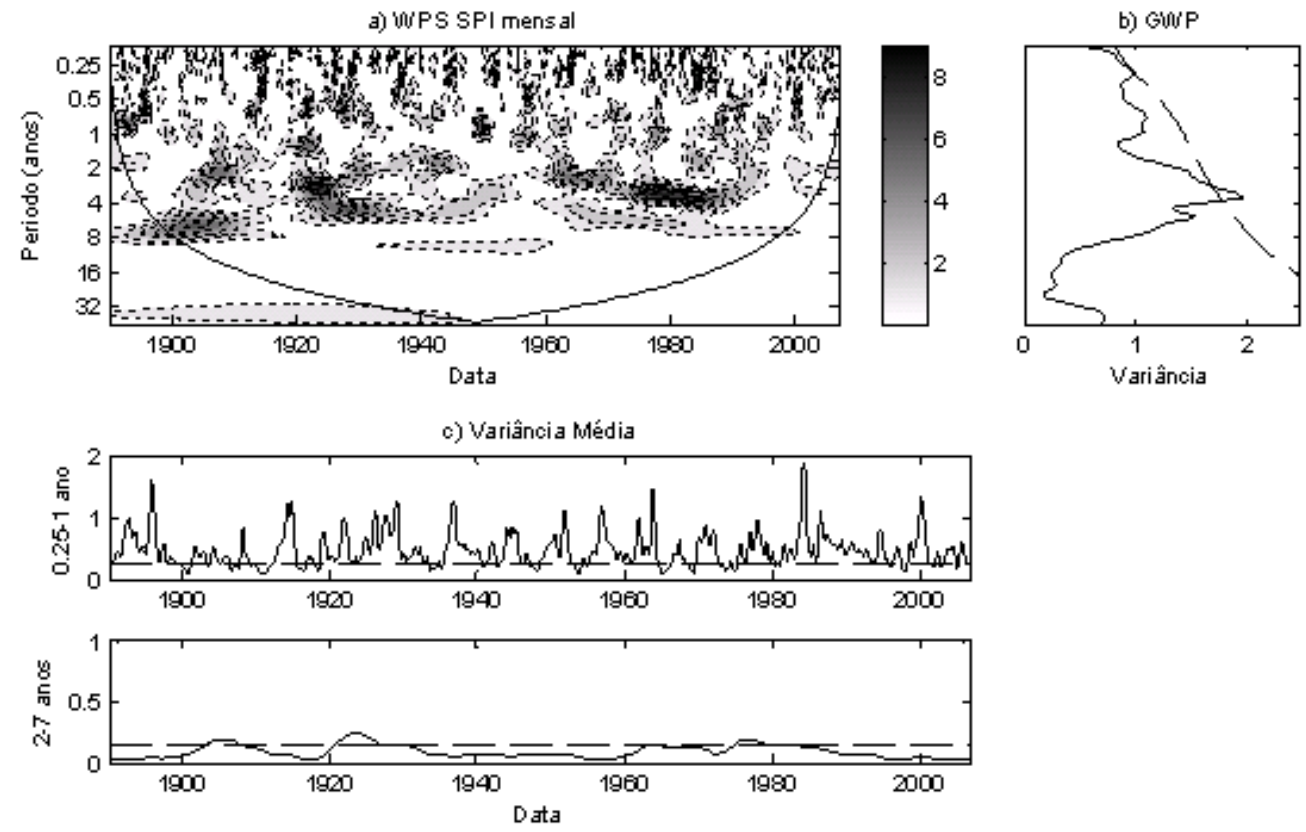

Figura 4 - a)Espectro de energia local da ondaleta o Índice Padronizado de Precipitação (SPI) de Campinas-SP. Contornos tracejados correspondem a valores de variância normalizados variando de 1 a 10, conforme representado na barra horizontal. Contornos sombreados englobam áreas com variâncias significativas ao nível de $95 \%$ de confiança. A curva em forma de U representa o cone de influência, sob a qual o efeito de borda é importante; b) Espectro global de ondaleta; c) média por escalas das potências observadas. 
De forma geral, a premissa de independência temporal relativa aos dados das séries mensais do SPI pode ser satisfatoriamente aceita, uma vez que apenas em dois casos (Tabela 2) o limite do ruído branco foi ultrapassado. Com isso pode-se admitir que as exigências necessárias à aplicação do MK são satisfeitas. Observando a Tabela 2, verifica-se que o mês de agosto é o único em que é verificado valor significativo, a $10 \%$ de probabilidade de rejeição de Ho verdadeira, do MK. Nesse aspecto, é interessante ressaltar o trabalho de Blain et al. (2007) que analisou a série decendial da razão entre a evapotranspiração real e evapotranspiração potencial (ETR/ETP) em Campinas (1890 a 2005). Esse quociente utilizado por Blain et al. (2007) pode, de forma simplista, ser entendido como a relação entre a demanda hídrica atmosférica, considerando apenas o balanço vertical de energia sobre uma cultura padrão, e a água realmente evapotranspirada por essa superfície padrão. Conceitualmente, quando não há deficiência hídrica no sistema considerado, a ETR/ETP atinge seu valor máximo igualando-se a 1. Segundo Blain et al. (2007) foi observada tendência de queda nos valores de ETR/ETP relativos aos decêndios do mês de agosto nos últimos 29 anos da série utilizada. A tendência de elevação nos déficits de PRE, indicada pelo valor negativo do MK do mês de agosto, pode ser vista como uma das causas da intensificação das condições de deficiência hídrica no solo, observada por Blain et al. (2007) para os decêndios do mês sob investigação.
Observa-se também, que o valor positivo mais elevado do MK ocorreu no mês de maio.

Quando aplicado para toda a série do SPI, temporalmente ordenada, o valor do MK anual, -1,30, também não indicou tendência significativas. Na Figura 6 é ilustrada a série temporal do SPI mensal na localidade de Campinas, entre 1890 e 2007, que ilustra claramente a ausência de tendência de longo prazo.

\section{CONCLUSÃO}

A série mensal do Índice Padronizado de Precipitação pode ser considerada, ao nível de significância de $5 \%$, livre de persistência temporal. Essa característica pode ser vista como conseqüência esperada da elevada variabilidade temporal dos dados de precipitação pluvial da série de Campinas-SP e do próprio algoritmo de cálculo do índice de seca empregado.

A análise de ondaleta indicou uma variabilidade na escala de três a quatro anos, nos picos de potência da série temporal do SPI. Esses picos foram especialmente devido a variabilidades localizadas nos anos de 1920 a 1940 e 1970 a 1990. Apesar dessa última característica, não foi possível verificar diferença de fase consistente entre o IOS e o SPI. Portanto, o fenômeno ENOS não tem uma influência marcada nas condições mensais de variabilidade climática na localidade de Campinas. Contudo, os sinais espectrais, observados na referida escala temporal,

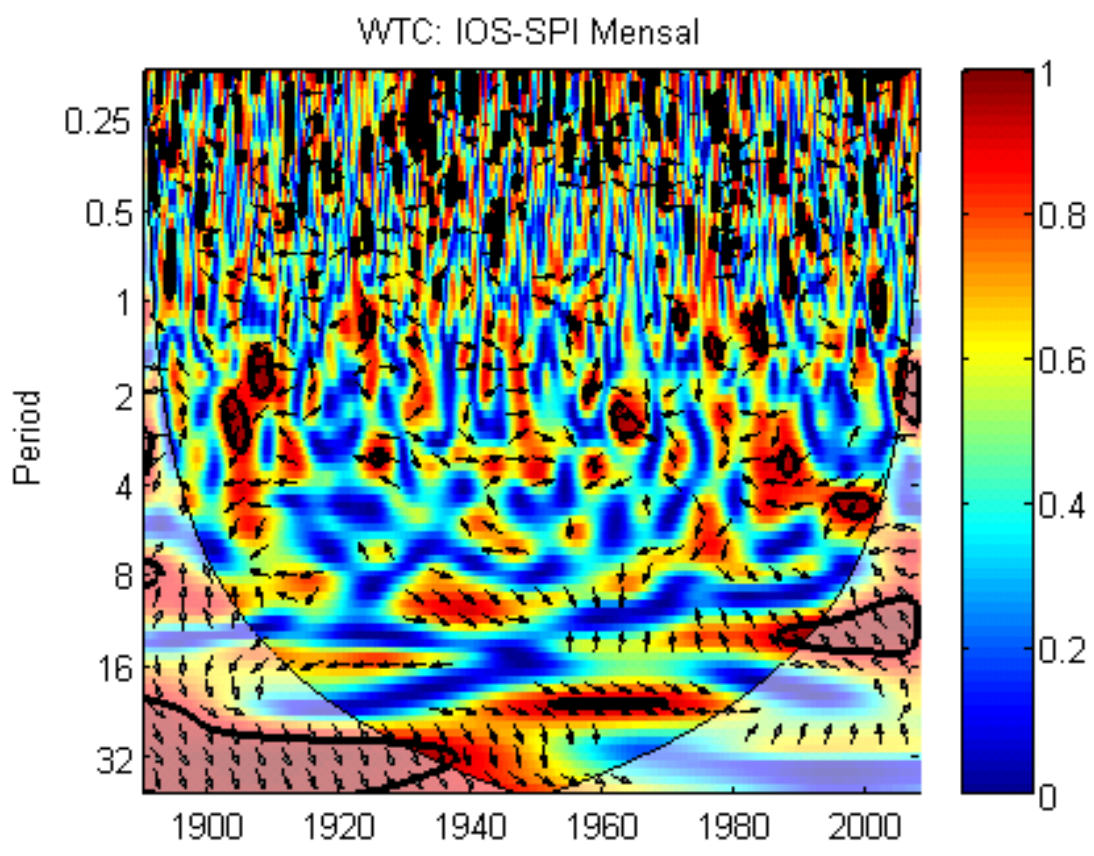

Figura 5 - Coerência e diferença de fase (indicadas por setas) do IOS e SPI em Campinas-SP. Linhas contínuas de cor preta indicam coerência significativa ao nível de 5\%. Apenas as setas correspondentes a coerência superior à 0,8 foram mostradas. A convenção das setas é a seguinte: horizontais para a direita indicam que as duas séries estão em fase; horizontais para esquerda indicam que as duas séries estão fora de fase; verticais para baixo indicam que a série de SPI está $90^{\circ}$ atrasada em relação à do IOS; verticais para cima indicam que a série de SPI está $90^{\circ}$ graus adiantada 
poderão, em trabalhos futuros, serem correlacionados com séries temporais da atividade solar que, por sua vez, possuem ciclos de 11 anos, com variações entre 8 a 15 anos. No que concerne às tendências de longo prazo, no mês de agosto foi detectada uma tendência de intensificação nas condições de déficit de precipitação pluvial. Nos demais meses não houve alterações significativas.

A capacidade de padronizar déficits/excessos de precipitação pluvial oriundos de distribuições com formas ou escalas consideravelmente distintas, além de conferir ao SPI uma importante característica no monitoramento e, conseqüentemente, na mitigação do fenômeno seca, torna esse índice uma interessante alternativa na investigação de forçantes condicionantes/moduladoras do clima, especialmente as relacionados ao evento seca, de determinada região.

Tabela 2 - Aplicação do teste não paramétrico de Mann-Kendal (MK) e da função auto-correlação (acf) nas séries mensais do Índice Padronizado de Precipitação na série de Campinas, estado de São Paulo (1890 a 2007)

\begin{tabular}{|c|c|c|c|c|c|c|c|}
\hline Mês & MK & \multicolumn{7}{|c|}{ Acf: limites ruído branco [-0,180:0,180] } \\
\hline janeiro & 0,51 & $-0,131$ & $-0,002$ & 0,025 & $-0,083$ & $-0,099$ & $-0,038$ \\
\hline fevereiro & $-0,87$ & $-0,022$ & 0,022 & 0,043 & $-0,037$ & 0,1 & 0,032 \\
\hline março & $-0,16$ & 0,028 & $-0,147$ & 0,067 & 0,16 & $0,241^{*}$ & $-0,055$ \\
\hline abril & 0,59 & $-0,096$ & $-0,075$ & 0,147 & 0,014 & $-0,121$ & $-0,068$ \\
\hline maio & 1,28 & 0,114 & $-0,054$ & $-0,022$ & 0,094 & $-0,091$ & 0,081 \\
\hline junho & $-0,8$ & $-0,177$ & 0,091 & $-0,161$ & 0,061 & $-0,13$ & 0,079 \\
\hline julho & 0,74 & 0,019 & $-0,124$ & 0,048 & $-0,02$ & $-0,12$ & 0,029 \\
\hline agosto & $-1,67$ & $-0,124$ & 0,093 & $-0,028$ & 0,153 & $-0,096$ & $-0,03$ \\
\hline setembro & $-0,82$ & $-0,024$ & $-0,099$ & $-0,028$ & 0,03 & 0,009 & 0,123 \\
\hline outubro & 0,06 & 0,106 & $-0,165$ & 0,105 & $-0,048$ & $-0,043$ & $-0,019$ \\
\hline novembro & $-0,93$ & $-0,045$ & $-0,061$ & $-0,056$ & 0,04 & $-0,196^{*}$ & 0,142 \\
\hline dezembro & $-0,25$ & $-0,12$ & 0,091 & $-0,178$ & 0,11 & 0,07 & 0,122 \\
\hline
\end{tabular}

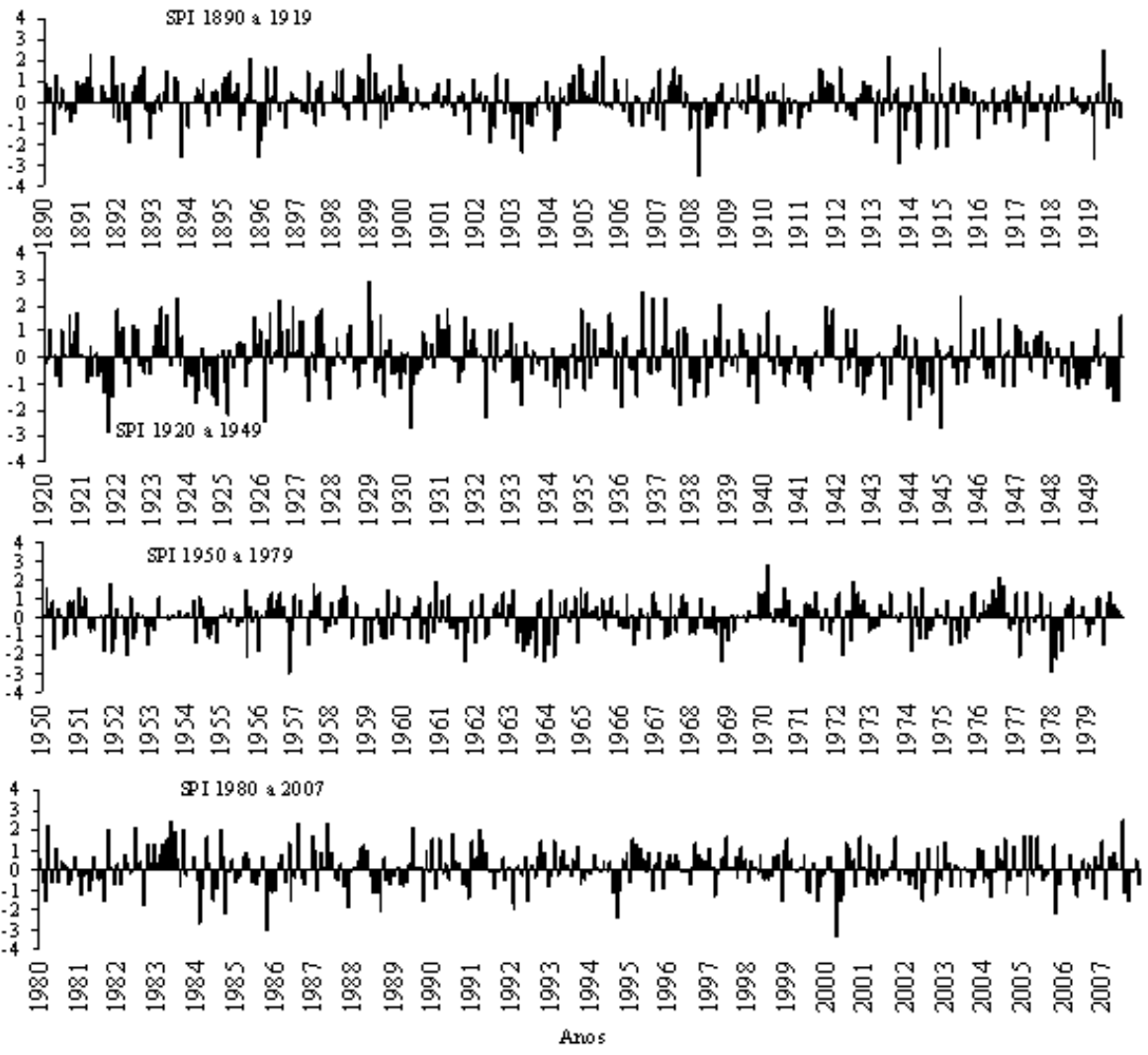

Figura 6 - Coerência e diferença de fase (indicadas por setas) do IOS e SPI em Campinas-SP. Linhas contínuas de cor preta indicam coerência significativa ao nível de 5\%. Apenas as setas correspondentes a coerência superior à 0,8 foram mostradas. A convenção das setas é a seguinte: horizontais para a direita indicam que as duas séries estão em fase; horizontais para esquerda indicam que as duas séries estão fora de fase; verticais para baixo indicam que a série de SPI está $90^{\circ}$ atrasada em relação à do IOS; verticais para cima indicam que a série de SPI está $90^{\circ}$ graus adiantada 


\section{AGRADECIMENTOS}

Os autores agradecem aos dois revisores pelas valiosas sugestões que contribuíram para a melhoria do texto final. A análise cruzada de ondaletas foi aplicada utilizando o algoritmo elaborado por Aslak Grinsted - Copyright (C) 20022004 - disponível em http://www.pol.ac.uk/home/research/ waveletcoherence/na linguagem utilizada pelo software Matlab. Os autores agradecem esse pesquisador pela disponibilização do algoritmo.

\section{REFERÊNCIAS}

ABRAMOWITZ, M.; STEGUN., I.A. Handbook of mathematical function, Dover, pp.1046, 1965.

BLAIN, G. C. Avaliação e adaptação do Índice de Severidade de Seca de Palmer (PDSI) e do Índice Padronizado de Precipitação (SPI) às condições climáticas do Estado de São Paulo, 2005, 120p, Dissertação (Mestrado em Agricultura Tropical e Sub-tropical) - Instituto Agronômico, Campinas - SP.

BLAIN, G. C.; PIEDADE, S. M. S.; CAMARGO, M. B. P.; GIAROLLA, A. Distribuição temporal da precipitação pluvial mensal observada no Posto Meteorológico do Instituto Agronômico, em Campinas, SP. Bragantia, v. 66, n.2, p. 347-355, 2007.

BLAIN, G.C.; CAMARGO, M.B.P.; PEREIRA, A.R. Detection of possible alterations (trends) in the ten day period ratio between actual and potential evapotranspiration during the years 1890 to 2005 in the region of CampinasSP-Brazil. In: CONGRESSO BRASILEIRO DE AGROMETEOROLOGIA, 15, Aracajú. 2007 Anais. Sociedade Brasileira de Agrometeorologia, (CDROM).

BLAIN, G. C. Considerações estatísticas relativas à oito séries de precipitação pluvial da Secretaria de Agricultura e Abastecimento do Estado de São Paulo, Revista Brasileira de Meteorologia, São José dos Campos, v.24, n. 1 p.12-23, 2009

BLAIN, G. C.; KAYANO, M. T.; CAMARGO, M. B. P.; LULU, J. Variabilidade amostral das séries mensais de precipitação pluvial em duas regiões do Brasil: Pelotas-RS e Campinas-SP Revista Brasileira de Meteorologia, São José dos Campos, v.24, n. 1 p.1-11, 2009

CUNHA, G.R.; DALMAGO, G.A.; ESTEFANEL, V. ENSO influences on wheat crop in Brazil. Revista Brasileira de Agrometeorologia, Santa Maria, v.7, n.1, p.127-138, 1999.

CUNHA, G.R.; DALMAGO, G.A.; ESTEFANEL, V. et al. El Niño - Oscilação do Sul e seus impactos sobre a cultura de cevada no Brasil. Revista Brasileira de Agrometeorologia, Santa Maria, v.9, n.1, p.137-145, 2001.
GRINSTED, A.; MOORE, J. C.; JEVREJEVA, S. Application of the cross wavelet transform and wavelet coherence to geophysical time series, Nonlinear processes in geophysics, v.11, p. 561-566, 2004

GUEDES, R. L.; ANDREOLI, R. V.; KAYANO, M.T.; OYAMA, M. D.; ALVES, M. A. S. Série temporal de precipitação mensal de Fortaleza, Brasil: Comparação entre observações e dados de reanálise do NCEP/NCAR, Revista Brasileira de Meteorologia, São José dos Campos, v.20, n. 1 p.83-92, 2005

GUTTMAN, G.B. Comparing the Palmer Drought Index and the "Standardized Precipitation Index". Journal of the American Water Resources, Washington, v.16, n.2, p. 113-121, 1998.

GUTTMAN, G.B. Accepting the "Standardized Precipitation Index": A calculation algorithm Index. Journal of the American Water Resources, Washington, v.35, n.2, p. 311-322, 1999.

HAYES, M. J.; SVOBODA, M.D.; WILHITE, D.A.; VANYARKHO, O.V. Monitoring the 1996 drought using the Standardized Precipitation Index. Bulletin of the American Meteorological Society. v. 80, n.3, p.429-438, 1999.

HEIM JUNIOR, R. R, A review of twentieth - century drought indices used in the United States. Bulletin of the American Meteorological Society, v.83, n.8, p.1149-1163, 2002.

IPCC, Climate Change 2001: Impacts, Adaptation and Vulnerability, Contribution of Working Group 2 to the Third Assessment Report of the Intergovernmental Panel on Climate Change, HOUGHTON, JT, Cambridge University Press, 2001.

KAYANO, M. T.; BLAIN, G. C. Análisis de ondeletas de series mensuales de precipitación, temperaturas máximas y mínimas mensuales en Campinas. In: CONGRESO VENEZOLANO DE AGROMETEOROLOGIA, 4. 2007. Maracay, Anais Fundacite Táchira

KENDALL, M. A.; STUART, A. The advanced theory of statistics, Londres: Charles Griffin \& Company Limited, 1967 v2 ed 2, 690p.

LI, W.; FU, R.; JUAREZ, R.I.N.; FERNANDES, K. Observed change of the standardized precipitation index, its potential cause and implications to future climate change in the Amazon region, Philosophical Transactions of the Royal Society, v. 363, p.1767-1772, 2008 doi:10.1098/rstb.2007.0022

MANN, H.B. Non-parametric tests against trend, Econometrica 13, MathSciNet, 1945, pp. 245-259.

MARENGO, J.; NOBRE, C.; RAIGOZA, D.; VALVERDE, M.; PISNITCHENKO, I.A.; OLIVEIRA, J.C.M. Boletim do Projeto: uso de cenários de mudanças climáticas regionais em estudos de vulnerabilidade e adaptação no Brasil e na América do Sul (GOF-UK-CPTEC), 2007. 
Disponível em: <www.cptec.inpe.br/mudancas_climaticas $>$. Acesso em: 1 nov. 2008.

MCKEE, T.B.; DOESKEN, N.J.; KLEIST, J. The relationship of drought frequency and duration to times scale. In: Conference on Applied Climatology, 8.,1993, Boston. Anais... American Meteorological Society, Boston: PREPRINTS, p.179 - 184, 1993

MCKEE, T.B.; DOESKEN, N.J. e KLEIST,J. Drought monitoring with multiple times scales. In: CONFERENCE ON APPIIED CLIMATOLOGY, 9.,1995, Boston. Anais... American Meteorological Society, Boston: PREPRINTS, p.233-236, 1995.

MELlO, M.H.; ALFONSI, R.R.; CAMARGO, M.B.P. Oscilações sul e o regime pluvial numa localidade do sudeste brasileiro. In: CONGRESSO BRASILEIRO DE AGROMETEOROLOGIA, 11., 1999, Florianópolis, SC. Anais..., Florianópolis: Sociedade Brasileira de Agrometeorologia/ EPAGRI, 1999a. 631p. 7p. (CD-ROM).

MELlO, M.H.; CAMARGO, M.B.P.; ALFONSI, R.R Contribuição ao estudo dos efeitos das oscilações sul no comportamento térmico, em localidades tropicais. In: CONGRESSO BRASILEIRO DE AGROMETEOROLOGIA, 11., 1999, Florianópolis, SC. Anais..., Florianópolis: Sociedade Brasileira de Agrometeorologia/ EPAGRI, 1999b. 631p. 6p. (CD-ROM).

MINUZZI, R.B.; SEDIYAMA, G. C.; COSTA, J. M. N.; VIANELLO, R. L. Influência da La Niña na estação chuvosa da Região Sudeste, Revista Brasileira de Meteorologia, São José dos Campos, v.22, n. 3 p.345-353, 2007

MONTEIRO, C. A. F. A dinâmica climática e as chuvas no estado de São Paulo: estudo geográfico sob forma de atlas Universidade de São Paulo, Instituto de Geografia, 1973. São Paulo: p.154

MORETIIN, P.A. Análise de ondaletas. In: Ondas e Ondaletas: Da análise de Fourier à análise de ondaletas. São Paulo: Edusp, $1^{\text {nd }}$ ed., 1999, p.191-232

NAKKEN, M. Wavelet analysis of rainfall-runoff variability isolating climatic from anthropogenic patterns.

Environmental Modelling \& Software, v. 14, p. 283295, 1998.

NUNES, L.H. Distribuição espaço-temporal da pluviosidade no Estado de São Paulo: variabilidade, tendências, processos intervenientes. 1997. 192 f. Tese (Doutorado) Universidade Estadual de São Paulo, São Paulo.

PALMER, W.C. Meteorological drought. US Weather Bureau Res. $\mathbf{N}^{\mathbf{0}}$ 45. Washington, 58p, 1965.

REBOITA, M. S. Elementos da Variabilidade Climática no Extremo Sul do Brasil, no Período de 1990 a 2001, 2004, 211p, Dissertação (Mestrado em Engenharia Oceânica) Universidade Federal do Rio Grande do Sul, Rio Grande-RS.
RAO, V. B.; HADA, K. An experiment with linear regression in forecasting of spring rainfall over south Brasil. Atmósfera, v. 7, n. 2, p. $83-87,1994$.

ROPELEWSKY C.F.; HALPERT, M.S. Global and regional scale precipitation patterns associated with the El Niño Southern Oscillation. Monthly Weather Review, Boston, v.115, n.1, p.1606-1626, 1987.

ROPELEWSKY C.F.; HALPERT, M.S. Precipitation patterns associated with the high index phase of the Southern Oscillation. Journal of Climate, Boston, v.2, p.268-284, 1989.

SANSIGOLO, C.A. Análise comparativa do desempenho dos Índices de Seca de Palmer (PDSI) e da Precipitação Normalizada (SPI) em Piracicaba, SP (1917 - 2001). Revista Brasileira de Meteorologia, Santa Maria, v.19, n.1, p. 51-56, 2004.

TADESSE, T.; WILHITE, D.A.;MICHAEL J. HAYES, M.J. Discovering Associations between Climatic and Oceanic Parameters to Monitor Drought in Nebraska Using DataMining Techniques, Journal of Climate, Boston, v.18, p.1541-1550, 2005

THOM, H. C. S. Same methods of climatological analyses. World Meteorological Organization, Geneva, 53p, 1966. (WMO, 199; TP, 103; Technical note. ,81.)

THORNTHWAITE, C.W., MATHER, J.R. The water balance. Climatology, v.8, n.1, p.1 - 40, 1955.

TORRENCE, C; COMPO, G.P. A Practical Guide to Wavelet Analysis, Bulletin of American Meteorological Society, 79, 61-78. 1998

TORRENCE, C.; WEBSTER, P. Interdecadal changes in the ENSO-monsoon system, Journal of Climate., v.12, p.2679 $-2690,1999$.

WILHITE, D. A.; GLANTZ, M.H. Understanding the drought phenomenon: The role definations. In: WILHITE et al. Planning for drought toward a reduction of societal vulnerability. WESTVIEW, 1987. p. 11-14.

WILHITE, D. A. Drought as a natural hazard: Conceptions and definitions. In: WILHITE, D. A. Drought : A global assessment. Routledge, 2000. p. $111-120$.

WILKS, D.S. Theoretical probability distributions, In: Statistical methods in the atmospheric sciences, San Diego: Academic Press, $2^{\text {nd }}$ ed, 2006, p, 102-104.

ZHAI, J.; SU, B.; KRYSANOVA, V.; TOBIAS, VETTER, T.; GAO, C.; JIANG, T. Spatial variation and trends in PDSI and SPI indices and their relation to streamflow in ten large regions of China, Journal of Climate, v.23, n.3, p. 649663, 2010 